\begin{tabular}{|c|c|}
\hline Title & Design of a reflection-suppressed all-optical diode based on asymmetric L-shaped nonlinear photonic crystal cavity \\
\hline Author(s) & Sato, Takanori; Makino, Shuntaro; Fujisawa, Takeshi; Saitoh, Kunimasa \\
\hline Citation & $\begin{array}{l}\text { Journal of the Optical Society of A merica B, 33(1), } 5461 \\
\text { https://doi.org/10.1364/JOSA B.33.000054 }\end{array}$ \\
\hline Issue Date & 2016-01-01 \\
\hline Doc URL & http:/hdl .handle.net/2115/67119 \\
\hline Rights & $\begin{array}{l}\text { (1) } 2016 \text { Optical Society of A merica. One print or electronic copy may be made for personal use only. Systematic } \\
\text { reproduction and distribution, duplication of any material in this paper for a fee or for commercial purposes, or } \\
\text { modifications of the content of this paper are prohibited. }\end{array}$ \\
\hline Type & article (author version) \\
\hline File Information & 250992.pdf \\
\hline
\end{tabular}

Instructions for use 


\title{
Design of reflection-suppressed all-optical diode based on asymmetric L-shaped nonlinear photonic crystal cavity
}

\author{
TAKANORI SATO,* Shuntaro Makino, TAKeshi FujISAWA, AND KunimaSA \\ SAITOH \\ Graduate School of Information Science and Technology, Hokkaido University North 14, West 9, Kita-ku, Sapporo 060-0814, Japan \\ *Corresponding author: tsato@icp.ist.hokudai.ac.jp
}

Received XX Month XXXX; revised XX Month, XXXX; accepted XX Month XXXX; posted XX Month XXXX (Doc. ID XXXXX); published XX Month XXXX

A simple design method for suppressing the reflection of the all-optical diode based on the L-shaped photonic crystal (PC) cavity is proposed. Analyzing the linear resonant characteristics of the PC cavity and using the nonlinear coupled-mode theory (NL-CMT), the strategy for obtaining reflection-suppressed structure is illustrated. Based on the design rule, "reflection-suppressed all-optical diode" is presented and numerical solutions indicate that the designed structure has very small reflection as well as extremely large nonreciprocal transmission ratio (NTR).

OCIS codes: (190.0190) Nonlinear optics; (190.3270) Kerr effect; (230.1150) All-optical devices; (130.5296) Photonic crystal waveguides.

http://dx.doi.org/10.1364/AO.99.099999

\section{INTRODUCTION}

Nonreciprocal optical devices, such as optical isolators and optical diodes, are crucial components in optical communication system. For example, an optical isolator protects lasers from back reflections. An optical diode is a nonreciprocal two-port device that transmits forward propagating light and do not transmit backward propagating light. To realize the nonreciprocal diode operation, it is necessary to break the Lorentz reciprocity [1-3]. Although magneto-optical materials, including garnet, have nonreciprocal property, such materials are unfortunately incompatible with a silicon-on-insulator (SOI) platform $[4,5]$. Another approach to realize the diode operation is to use nonlinear optical effects, such as optical Kerr-effect, free-carrier effect, thermo-optic effect, four-wave mixing, stimulated Brillouin scattering, and so on [6-15]. These optical diodes based on the optical nonlinear effects have "dynamic reciprocity", and hence the diode operation can be observed under certain input conditions [2,3]. To achieve the diode operation based on the intensity-dependent refractive index, the structural asymmetry is indispensable for the symmetry breaking of the transmission characteristics. So far, two types of all-optical nonlinear diodes based on the intensity-dependent refractive index have been proposed. In [11-13], the nonlinear optical diodes are composed of two different resonators, and the transmission is estimated by the product of two nonlinear transmission spectra. However, the overall structure is relatively large. On the other hand, the nonlinear optical diodes composed of a single photonic crystal (PC) cavity that has two asymmetric input and output ports are superior in terms of structural miniaturization $[7-9,15]$. Recently, it was reported that the asymmetric
PC cavity can exhibit the nonreciprocal transmission by its nonlinear mode coupling and perform the nonreciprocal transmission ratio (NTR) of $50 \mathrm{~dB}$ [15]. NTR is defined as the ratio of the backward transmission to forward transmission and used to evaluate the optical nonreciprocity. In the ideal case, the forward transmission of $100 \%$ and backward transmission of $0 \%$ are desired as shown in Fig. 1 (a) and (b), resulting in NTR of $+\infty$. To the best of our knowledge, the theoretically and experimentally reported maximum values of NTR are $50 \mathrm{~dB}$ [15] and 40 $\mathrm{dB}[16]$, respectively. Although it was not fully pointed out in previous

(a) Ideal Forward Propagation

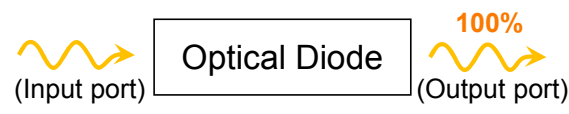

(b) Ideal Backward Propagation

$0 \%$

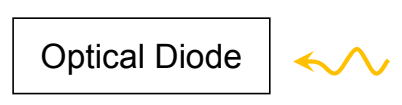

(c) Undesired Back Reflection for Forward Propagation

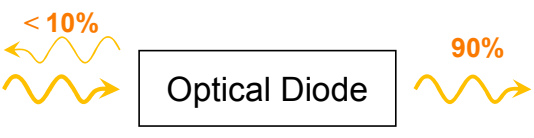

Fig. 1. Illustration of the optical diode. (a) Ideal forward propagation. (b) Ideal backward propagation (c) Nonideal forward propagation due to the existence of back reflection. 
literatures, in addition to NTR, the suppression of back reflections is also an important feature. As shown in Fig. 1(c), even if NTR is $+\infty$, the reflection should be suppressed especially for PC-type optical diodes since light cannot be radiated to the cladding. As discussed above, the low-reflection characteristics are remarkably important for the alloptical diode as well as the large NTR value. For the L-shaped nonlinear PC cavity [15], both large NTR and low reflection can be easily obtained if the reflection-suppressed structure can be designed. The nonlinear characteristics of the all-optical diode can be investigated by using the two-dimensional (2D) finite-element time-domain beam propagation method (FETD-BPM) [17] for the nonlinear optical waveguide [18], called as "NL-FETD-BPM" which can obtain nonlinear solutions rigorously. However, a lot of calculation time is required for specifying the reflection-suppressed structure and input conditions by trial and error. Hence, the strategy for suppressing the reflection is essential for designing the all-optical diode based on the L-shaped nonlinear PC cavity.

In this paper, the design rule for suppressing the reflection of twoport nonlinear optical diode based on the asymmetric L-shaped nonlinear PC cavity is presented. By using the combination of the nonlinear coupled-mode theory (NL-CMT) $[15,19,20]$ and FETDBPM [17], it is possible to specify the reflection-suppressed structure. The brief formulation of both methods is provided in Section 2. In the following section, the design method to suppress the reflection on the diode operation is illustrated and it is shown that only two parameters are crucial to determine the reflection characteristics. And then, the nonlinear characteristics of the designed all-optical diode are examined by NL-FETD-BPM [18]. The results obtained by NL-FETD-BPM agree well with those obtained by the proposed method, showing the validity of the discussion.

\section{NONLINEAR ANALYTICAL METHODS}

\section{A. Coupled-Mode Theory}

Figure 2(a) shows the schematics of the all-optical diode based on the asymmetric L-shaped square lattice PC cavity, which is composed of the input and output ports and the nonlinear defect rod cavity (filled orange circle). The defect cavity supports two eigenfield distributions, called as "dipole modes". The forward lightwave propagating in the input port can couple to one dipole mode (labeled as "1st cavity mode") and the backward lightwave propagating in the output port can similarly couple to another dipole mode (labeled as "2nd cavity mode") as shown in Fig. 2(b). In the linear regime, two dipole modes can hardly couple each other due to their orthogonal eigenfield distributions. However, the Kerr-nonlinearity in the defect rod enables to break the orthogonality, resulting in thenonlinear coupling of the dipole modes. In order to break the symmetry in the transmission characteristics, the three and four rods are placed adjacent to the nonlinear cavity rod for the input and output ports, respectively. Figure 2(b) shows the illustration of the principle of the diode operation. In the nonlinear regime, if the input power and frequency are properly set, forward lightwave propagating in the input port can pass through the defect rod cavity due to the nonlinear coupling between two dipole modes.

From the coupled-mode theory for the nonlinear dipole mode $[15,19,20]$, we obtain the following equations

$$
\begin{aligned}
& \frac{d A_{1}(t)}{d t}=-j\left(\omega_{1}-V_{11}-\frac{j}{\tau_{1}}\right) A_{1}+j V_{12} A_{2}+\sqrt{\frac{2}{\tau_{1}}} E_{\mathrm{fw}} e^{-j \omega t}, \\
& \frac{d A_{2}(t)}{d t}=-j\left(\omega_{2}-V_{22}-\frac{j}{\tau_{2}}\right) A_{2}+j V_{21} A_{1}+\sqrt{\frac{2}{\tau_{2}}} E_{\mathrm{bw}} e^{-j \omega t},
\end{aligned}
$$

where $A_{i}(i=1,2)$ is the amplitude of $i$-th cavity mode, $\omega_{i}(i=1,2)$ is the normalized resonant frequency of $i$-th cavity mode, $\omega$ is the normalized frequency of input lightwave, $\tau_{i}(i=1,2)$ is the normalized decay rate of $i$-th cavity mode, and $E_{\mathrm{fw}}$ and $E_{\mathrm{bw}}$ are the amplitudes of the electric field (a)

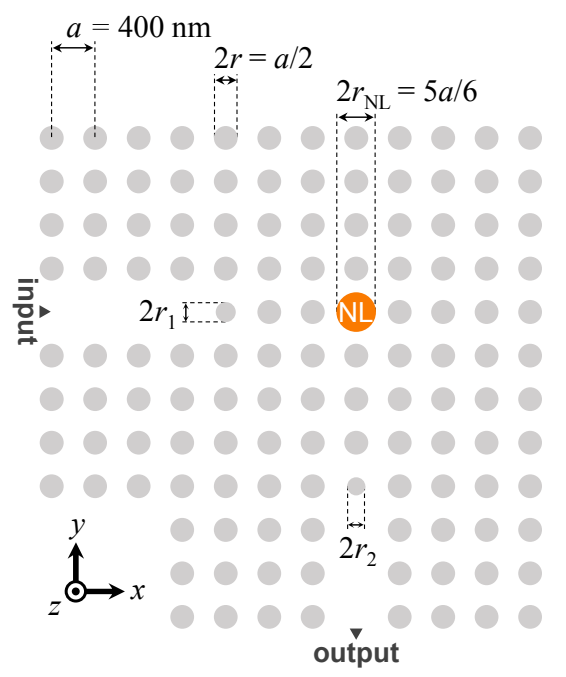

(b)

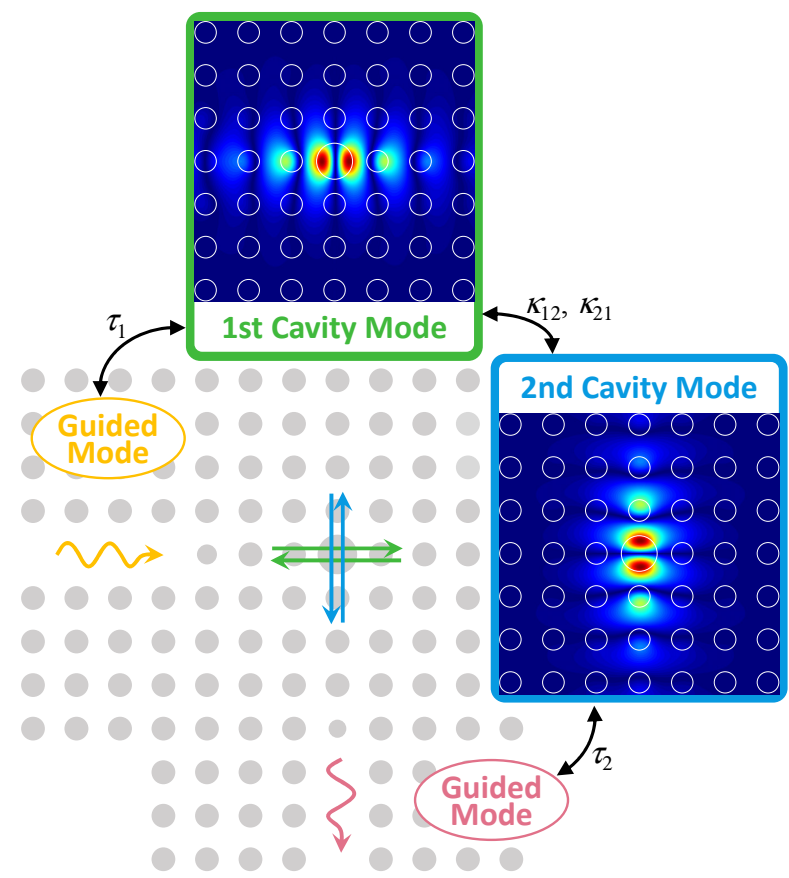

Fig. 2. (a) Schematics of all-optical diode based on the asymmetric Lshaped square lattice PC cavity and (b) illustration of the principle of the diode operation.

entering from the input and output ports, respectively. $\omega_{i}$ and $\tau_{i}$ are given by

$$
\begin{gathered}
\omega_{i}=\frac{a}{\lambda_{i}} \\
\tau_{i}=\frac{2 Q_{i}}{\omega_{i}}
\end{gathered}
$$

where $a$ is the lattice constant, $\lambda_{i}(i=1,2)$ is the resonant wavelength of $i$-th cavity mode, and $Q_{i}$ is $Q$-value of $i$-th cavity mode. The $Q$-value is defined as a ratio of resonant frequency to full width at half maximum. $V_{m n}(m, n=1,2)$ is the parameter of nonlinear contribution given by

$$
V_{m n}=\frac{\left(\omega_{m}+\omega_{n}\right)}{4 N_{m}} \int_{-\infty}^{+\infty} \int_{-\infty}^{+\infty} \delta \varepsilon(x, y) E_{m}(x, y) E_{n}(x, y) d x d y,
$$


where $N_{m}$ is the normalization constant, $\delta \varepsilon$ is the instantaneous Kerr change of the dielectric constant, and $E_{i}(i=1,2)$ is the electric field distribution of $i$-th cavity mode. $N_{m}$ and $\delta \varepsilon$ are defined as

$$
\begin{gathered}
N_{m}=\int_{-\infty}^{+\infty} \int_{-\infty}^{+\infty} n_{0}^{2}(x, y) E_{m}^{2}(x, y) d x d y=\frac{a^{2}}{c n_{2}}, \\
\delta \varepsilon \approx \frac{n_{0} c n_{2}\left|A_{1} E_{1}+A_{2} E_{2}\right|^{2}}{4 \pi},
\end{gathered}
$$

where $n_{0}$ is the linear refractive index, $n_{2}\left[\mathrm{~m}^{2} / \mathrm{W}\right]$ is the nonlinear index, $a$ is the lattice constant, and $c$ is the speed of light in vacuum. After substitution of Eqs. (5), (6), and (7) and $A_{m}=A_{m} e^{-j \omega t}$ into Eqs. (1) and (2), the stationary CMT $(\partial / \partial t \rightarrow-j \omega)$ can be derived as

$$
\begin{gathered}
\left(\omega-\omega_{1}+\kappa_{11}\left|A_{1}\right|^{2}+\kappa_{12}\left|A_{2}\right|^{2}+\frac{j}{\tau_{1}}\right) A_{1} \\
-2 \kappa_{12} \operatorname{Re}\left[A_{1}^{*} A_{2}\right] A_{2}=j \sqrt{\frac{2}{\tau_{1}}} E_{\mathrm{fw}}, \\
\left(\omega-\omega_{2}+\kappa_{22}\left|A_{2}\right|^{2}+\kappa_{21}\left|A_{1}\right|^{2}+\frac{j}{\tau_{2}}\right) A_{2} \\
-2 \kappa_{21} \operatorname{Re}\left[A_{1} A_{2}^{*}\right] A_{1}=j \sqrt{\frac{2}{\tau_{2}}} E_{\mathrm{bw}},
\end{gathered}
$$

where $\kappa_{m n}(m, n=1,2)$ is the nonlinear coupling coefficient for stationary CMT given by

$$
\kappa_{m n}=\frac{\left(\omega_{m}+\omega_{n}\right) n_{0} c^{2}}{16 \pi a^{2}} \int_{-\infty}^{+\infty} \int_{-\infty}^{+\infty} n_{2}^{2}(x, y) E_{m}^{2}(x, y) E_{n}^{2}(x, y) d x d y
$$

The transmission and back reflection of forward propagation (assuming $E_{\mathrm{bw}}=0$ ) light can be expressed as

$$
\begin{aligned}
& T_{\mathrm{fw}}=\frac{\left|\sqrt{\frac{2}{\tau_{2}}} A_{2}\right|^{2}}{\left|\sqrt{\frac{2}{\tau_{2}}} A_{2}\right|^{2}+\left|\sqrt{\frac{2}{\tau_{1}}} A_{1}-E_{\mathrm{fw}}\right|^{2}}, \\
& R_{\mathrm{fw}}=\frac{\left|\sqrt{\frac{2}{\tau_{1}}} A_{1}-E_{\mathrm{fw}}\right|^{2}}{\left|\sqrt{\frac{2}{\tau_{2}}} A_{2}\right|^{2}+\left|\sqrt{\frac{2}{\tau_{1}}} A_{1}-E_{\mathrm{fw}}\right|^{2}},
\end{aligned}
$$

and those of backward propagation can be expressed as well $[15,19,20]$.

\section{B. Finite-Element Time-Domain BPM}

For the analysis of the linear resonant characteristics of the PC cavity, the time-domain beam-propagation method (TD-BPM) based on the finite-element method (FEM), called as FETD-BPM, is used. FETD-BPM scheme is powerful numerical method to investigate the light propagation in the $\mathrm{PC}$ waveguides [17]. According to the procedures in the FETD-BPM, the time evolution of light propagation can be obtained by solving the following simultaneous linear equation

$$
[A(n(\mathbf{r}))]\{\phi\}_{i+1}=[B(n(\mathbf{r}))]\{\phi\}_{i},
$$

where $[A(n)]$ and $[B(n)]$ are the finite-element matrices related to refractive index distribution $n(\mathbf{r})$, and $\left\{\phi_{i}\right.$ and $\{\phi\}_{i+1}$ are electric or magnetic field vectors on $i$-th and $(i+1)$-th step.

By including the Kerr-nonlinearity in the material, we can solve nonlinear propagating problem as well. In the nonlinear regime, the refractive index can be expressed as

$$
n\left(\mathbf{r},|\mathbf{E}|^{2}\right)=n_{0}\left(1+\frac{1}{2} \varepsilon_{0} c n_{2}|\mathbf{E}|^{2}\right)
$$

where $\varepsilon_{0}$ is permittivity in vacuum, and $|\mathbf{E}|$ is the electric field vector. Substituting Eq. (14) to Eq. (13), the simultaneous equation for the nonlinear optical propagation analysis is derived as [18]

$$
\left[A\left(n\left(\mathbf{r},|\mathbf{E}|^{2}\right)\right)\right]_{i}\{\phi\}_{i+1}=\left[B\left(n\left(\mathbf{r},|\mathbf{E}|^{2}\right)\right)\right]_{i}\{\phi\}_{i},
$$

where $[A(n)]_{i}$ and $[B(n)]_{i}$ are $i$-th finite-element matrices whose nonlinear refractive index distribution is calculated by $\{\phi\}_{i}$. This method directly solves nonlinear Maxwell's equations rigorously and precise solutions can be obtained. Therefore, we use the results obtained by NLFETD-BPM to verify the validity of our proposed design.

\section{NUMERICAL ANALYSIS}

\section{A. Design and Basic Characteristics}

We assume the lattice constant $a=400 \mathrm{~nm}$, the radius of dielectric rod $r$ $=a / 4$, and the radius of defect rod $r_{\mathrm{NL}}=5 a / 12$ [21]. This PC structure has a photonic band gap (PBG) for the transverse-magnetic (TM) modes, which have electric field parallel to the rods, between $\omega_{\mathrm{min}}=0.244$ and $\omega_{\max }=0.292$. To introduce asymmetry, 3 and 4 rods are placed adjacent to the nonlinear rod for input and output ports. As seen in Fig. 2(a), the radii of two rods located at the edge of the input port and output port are assumed as $r_{1}$ and $r_{2}$ to control the resonant characteristics. The linear refractive indices of rods and background material are $n_{\text {rod }}=3.5$ and $n_{\text {back }}=1.5$, assuming the GaAs dielectric rods and silica glass, respectively [22]. We consider that the nonlinear index of defect rod is $n_{2}=1.5 \times 10^{-17} \mathrm{~m}^{2} / \mathrm{W}[23]$ and that of other materials is zero. The decay rate and resonant frequency of cavity modes are obtained by solving Eq. (13) [17].

We let start the case of $r_{1}=r_{2}=r$. Figure 3 shows the transmission spectra of 1st and 2nd cavity mode with $r_{1}=r_{2}=r$ and the relationship between resonant frequency and detuning parameter $\delta$ defined as $\left(\omega_{1}-\omega\right) \tau_{1} / 2$ [21]. Calculated resonant frequency and $Q$-value of 1 st and 2nd cavity modes are $\omega_{1}=0.26219$ and $Q_{1}=1280$, and $\omega_{2}=0.26224$ and $Q_{2}=5489$, respectively. Calculated nonlinear coupling coefficients are $\kappa_{11}=1.03 \times 10^{-4}, \kappa_{12}=3.75 \times 10^{-5}, \kappa_{21}=3.75 \times 10^{-5}$, and $\kappa_{22}=1.03 \times$

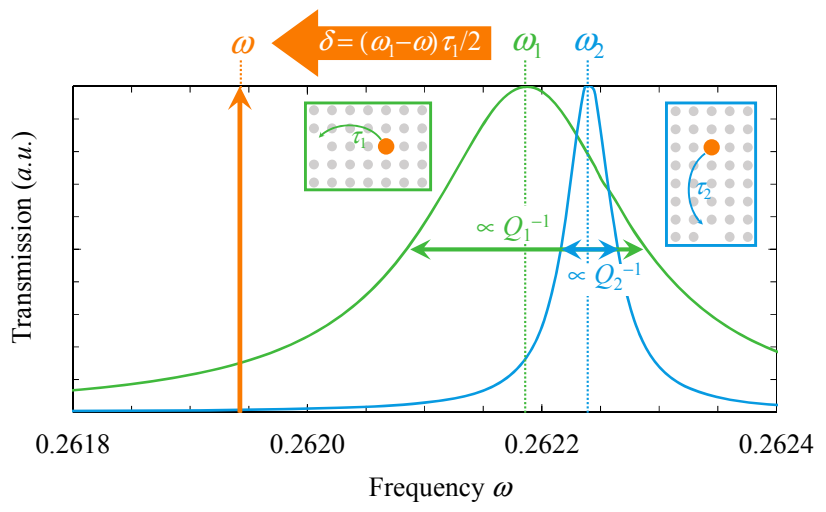

Fig. 3. Transmission spectra of 1 st and 2nd cavity mode with $r_{1}=r_{2}=r$ and the relationship between resonant frequency and detuning parameter $\delta(=2.39)$. 

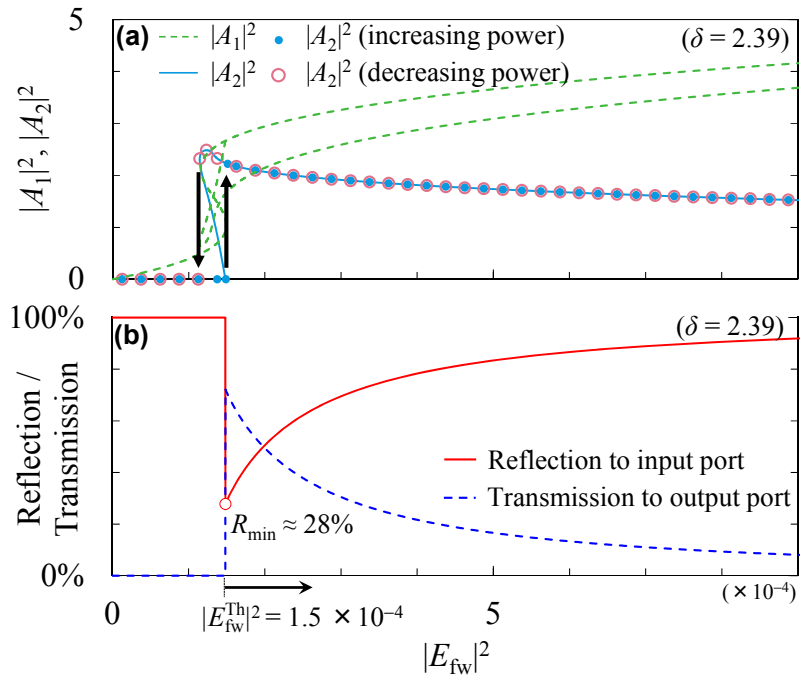

Fig. 4. Resonant characteristics of the all-optical diode with $r_{1}=r_{2}=r$ for the forward propagation ( $\delta=2.39$ ). (a) The amplitude of resonant modes (solid and dashed lines are calculated by Eqs. (8) and (9), and opened and closed circles are calculated by Eqs. (1) and (2) for increasing and decreasing $\left|E_{\mathrm{fw}}\right|$, respectively) and (b) reflection and transmission as a function of input intensity $\left|E_{\mathrm{fw}}\right|^{2}$.

$10^{-4}$. Figures 4 (a) and (b) show the resonant characteristics of the alloptical diode with $r_{1}=r_{2}=r$ for the forward propagation (assuming $E_{\mathrm{bw}}$ $=0)$ and the detuning parameter is $\delta=2.39(\omega=0.26194)$. The green dashed and blue solid lines in Fig. 4(a) represent the solutions of $\left|A_{1}\right|^{2}$ and $\left|A_{2}\right|^{2}$ calculated by the Eqs. (8) and (9). The closed and opened circles represents steady solutions of $\left|A_{2}\right|^{2}$ for increasing and decreasing power, respectively. Although the nonlinear interaction between dipole modes do not appear if input amplitude is sufficiently low, the both solutions $\left(\left|A_{1}\right|\right.$ and $\left.\left|A_{2}\right|\right)$ are simultaneously observed for the higher power due to the nonlinear coupling. As shown in Fig. 4(b), the minimum reflection for this structure is $R_{\min }=28 \%$ ( $-6 \mathrm{~dB}$ ). Figures $5(\mathrm{a})$ and (b) show the resonant characteristics of the all-optical diode with $r_{1}=r_{2}=r$ for the backward propagation (assuming $E_{\mathrm{fw}}=0$ ). The threshold intensity for the propagation and backward propagation is $\left|E^{\mathrm{Th}_{\mathrm{fv}}}\right|^{2}=1.5 \times 10^{-4}$ and $\left|E^{\mathrm{Th}} \mathrm{bw}\right|^{2}=5.1 \times 10^{-4}$. Therefore, the diode operation can be observed within the range of $\left|E_{\mathrm{fw}}\right|^{2}$ from $1.5 \times 10^{-4}$ to $5.1 \times 10^{-4}$ and $E^{\mathrm{Th}} \mathrm{fw}<E_{\mathrm{fw}}<$ $E^{\mathrm{Th}} \mathrm{bw}$ is necessary for the diode operation. Figure 6 shows the map of the reflection of the all-optical diode with $r_{1}=r_{2}=r$ as a function of input intensity $\left|E_{\mathrm{fw}}\right|^{2}$ and the detuning parameter $\delta$. In the area labeled as "Diode operation is impossible", the backward transmission is permitted when the lightwave enter from output port for the input power of $E_{\mathrm{fw}}$. The minimum reflection is $R_{\min }=28 \%$ when $\left|E_{\mathrm{fw}}\right|^{2}=$ $1.5 \times 10^{-4}$ and $\delta=2.39$ for the all-optical diode with $r_{1}=r_{2}=r$. To reduce the reflection further, the appropriate design method is required.

\section{B. Strategy for Suppressing the Reflection}

The resonant characteristics are determined by the following parameters: $\omega, \omega_{1}, \omega_{2}, \tau_{1}, \tau_{2}, \kappa_{11}, \kappa_{12}, \kappa_{21}$, and $\kappa_{22}$. Except for $\omega$, other parameters $\left(\omega_{i}, \tau_{i}\right.$, and $\left.\kappa_{m n}\right)$ are fixed by the structural parameters. Among these parameters, $\omega_{i}$, and $\tau_{i}$ can be easily controlled by $r_{i}$, whereas $\kappa_{m n}$, mainly defined by the eigenmode distribution, is hardly affected by $r_{i}$. Hence, we should focus on the following parameters: $\omega_{1} \omega_{1}, \omega_{2}, \tau_{1}$, and $\tau_{2}$.

Here, we consider following parameter transformation:

$$
\left(\begin{array}{lllll}
\omega & \omega_{1} & \omega_{2} & \tau_{1} & \tau_{2}
\end{array}\right) \rightarrow\left(\begin{array}{lllll}
\omega_{1} & Q_{1} & \delta & p_{1} & p_{2}
\end{array}\right)
$$

where $p_{1}=\tau_{2} / \tau_{1}$ and $p_{2}=\tau_{1}\left(\omega_{2}-\omega_{1}\right)$. Equations (8), (9), and (12) can be rewritten as
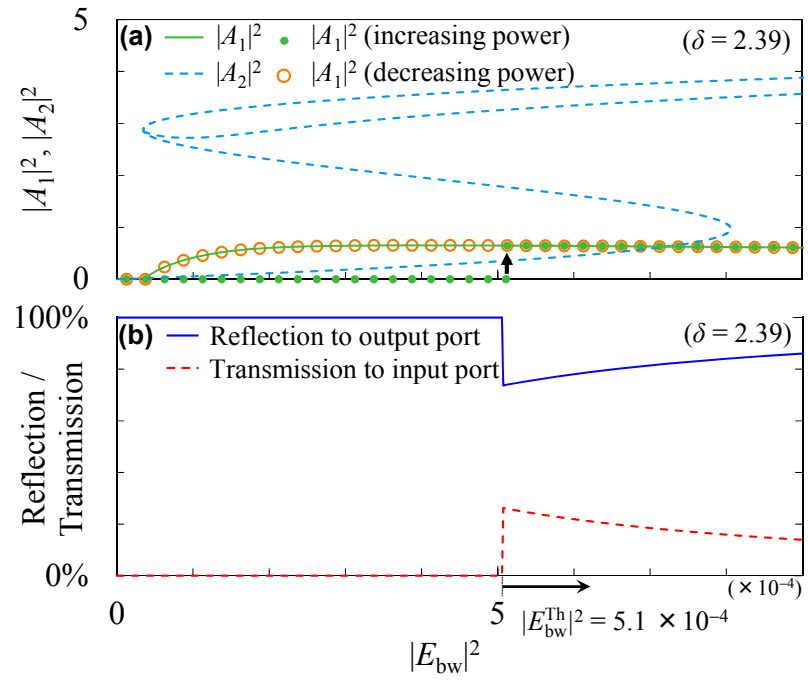

Fig. 5. Resonant characteristics of the all-optical diode with $r_{1}=r_{2}=r$ for the backward propagation ( $\delta=2.39$ ). (a) The amplitude of resonant modes (solid and dashed lines are calculated by Eqs. (8) and (9), and opened and closed circles are calculated by Eqs. (1) and (2) for increasing and decreasing $\left|E_{\mathrm{bw}}\right|$, respectively) and (b) reflection and transmission as a function of input intensity $\left|E_{\mathrm{bw}}\right|^{2}$.

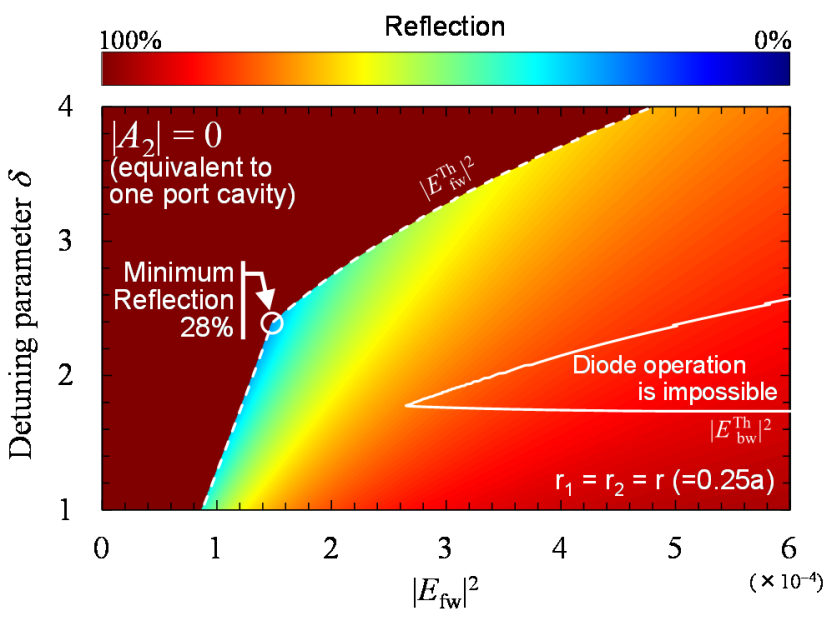

Fig. 6. Map of the reflection of the all-optical diode with $r_{1}=r_{2}=r$ for the forward propagation as a function of input power $\left|E_{\mathrm{fw}}\right|^{2}$ and the detuning parameter $\delta$.

$$
\begin{aligned}
& \left(-\delta \frac{\omega_{1}}{Q_{1}}-\kappa_{11}\left|A_{1}\right|^{2}-\kappa_{12}\left|A_{2}\right|^{2}+\frac{j}{2} \frac{\omega_{1}}{Q_{1}}\right) A_{1} \\
& -2 \kappa_{12} \operatorname{Re}\left[A_{1}^{*} A_{2}\right] A_{2}=j \sqrt{\frac{\omega_{1}}{Q_{1}}} E_{\mathrm{fw}}, \\
& \left(-\delta \frac{\omega_{1}}{Q_{1}}-\frac{p_{2}}{2} \frac{\omega_{1}}{Q_{1}}-\kappa_{22}\left|A_{2}\right|^{2}-\kappa_{21}\left|A_{1}\right|^{2}+\frac{j}{2 p_{1}} \frac{\omega_{1}}{Q_{1}}\right) A_{2} \\
& -2 \kappa_{21} \operatorname{Re}\left[A_{1} A_{2}^{*}\right] A_{1}=j \sqrt{\frac{1}{p_{1}} \frac{\omega_{1}}{Q_{1}}} E_{\mathrm{bw}} \text {, }
\end{aligned}
$$




$$
R_{\mathrm{fw}}\left(E_{\mathrm{fw}}\right)=\frac{\left|\sqrt{\frac{\omega_{1}}{Q_{1}}} A_{1}-E_{\mathrm{fw}}\right|^{2}}{\left|\sqrt{\frac{1}{p_{1}} \frac{\omega_{1}}{Q_{1}}} A_{2}\right|^{2}+\left|\sqrt{\frac{\omega_{1}}{Q_{1}}} A_{1}-E_{\mathrm{fw}}\right|^{2}} .
$$

If we define $\tilde{\omega}_{1}, \tilde{Q}_{1}$ as

$$
\frac{\tilde{\omega}_{1}}{\tilde{Q}_{1}}=\frac{\omega_{1}}{Q_{1}} \alpha
$$

with $\alpha$ being an arbitrary number, Eqs. (16) and (17) can be rewritten as

$$
\begin{gathered}
\left(-\delta \frac{\tilde{\omega}_{1}}{\tilde{Q}_{1}}-\kappa_{11}\left|\tilde{A}_{1}\right|^{2}-\kappa_{12}\left|\tilde{A}_{2}\right|^{2}+\frac{j}{2} \frac{\tilde{\omega}_{1}}{\tilde{Q}_{1}}\right) \tilde{A}_{1} \\
-2 \kappa_{12} \operatorname{Re}\left[\tilde{A}_{1}^{*} \tilde{A}_{2}\right] \tilde{A}_{2}=j \sqrt{\frac{\tilde{\omega}_{1}}{\tilde{Q}_{1}}} \tilde{E}_{\mathrm{fw}}, \\
\left(-\delta \frac{\tilde{\omega}_{1}}{\tilde{Q}_{1}}-\frac{p_{2}}{2} \frac{\tilde{\omega}_{1}}{\tilde{Q}_{1}}-\kappa_{22}\left|\tilde{A}_{2}\right|^{2}-\kappa_{21}\left|\tilde{A}_{1}\right|^{2}+\frac{j}{2 p_{1}} \frac{\tilde{\omega}_{1}}{\tilde{Q}_{1}}\right) \tilde{A}_{2} \\
-2 \kappa_{21} \operatorname{Re}\left[\tilde{A}_{1} \tilde{A}_{2}^{*}\right] \tilde{A}_{1}=j \sqrt{\frac{1}{p_{1}} \frac{\tilde{\omega}_{1}}{\tilde{Q}_{1}}} \tilde{E}_{\mathrm{fw}},
\end{gathered}
$$

where

$$
\begin{aligned}
& \tilde{E}_{\mathrm{fw}}=E_{\mathrm{fw}} \alpha, \\
& \tilde{E}_{\mathrm{bw}}=E_{\mathrm{bw}} \alpha, \\
& \tilde{A}_{1}=A_{1} \sqrt{\alpha}, \\
& \tilde{A}_{2}=A_{2} \sqrt{\alpha} .
\end{aligned}
$$

The reflection $\tilde{R}_{\mathrm{fw}}$ of the structure having $\tilde{\omega}_{1}$ and $\tilde{Q}_{1}$ as a function of $\tilde{E}_{\mathrm{fw}}$ is given by

$$
\tilde{R}_{\mathrm{fw}}\left(\tilde{E}_{\mathrm{fw}}\right)=R_{\mathrm{fw}}\left(E_{\mathrm{fw}}\right) .
$$

Resonant wavelength $(\lambda=a / \omega)[\mathrm{nm}]$

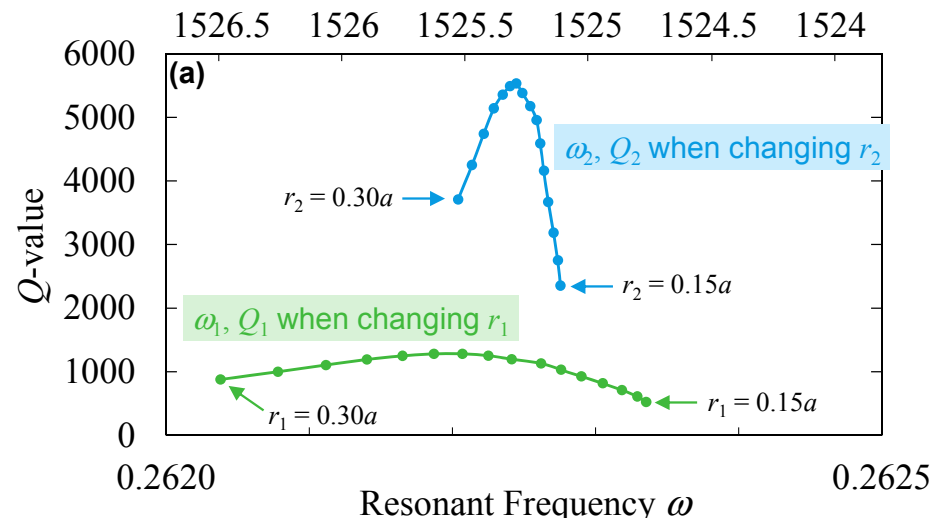

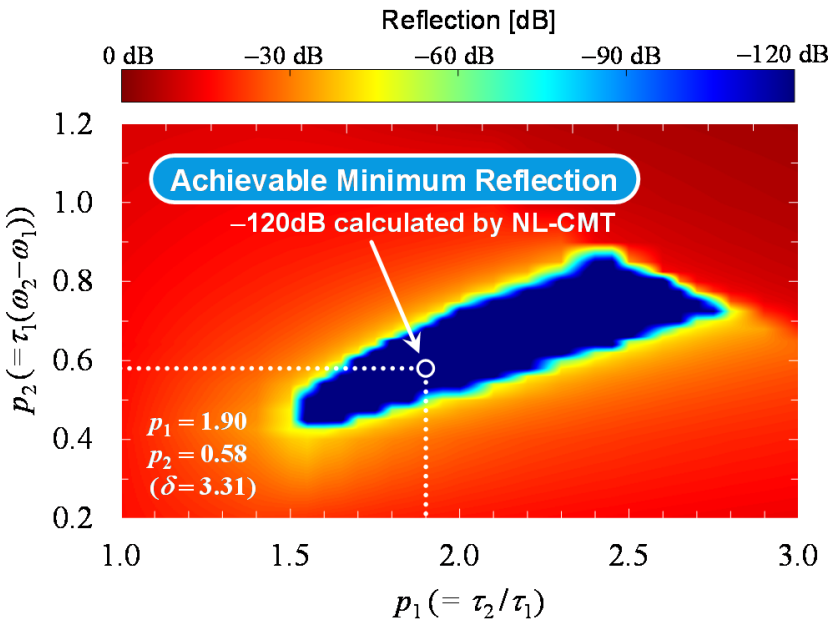

Fig. 7. Map of the minimum reflection of the all-optical diode as a function of two asymmetricity parameters for $\kappa_{1}=1.03 \times 10^{-4}, \kappa_{1}=3.75$ $\times 10^{-5}, \kappa_{21}=3.75 \times 10^{-5}$, and $\kappa_{22}=1.03 \times 10^{-4}$.

Equation (26) implies that, regardless of the ratio of $\omega$ and $Q_{1}$, the reflection is constant as long as $p_{1}, p_{2}$ and $\delta$ are fixed. Therefore, for searching the structure having minimum reflection, only $p_{1}, p_{2}$, and $\delta$ have to be considered. We can also choose $\omega_{2}$ and $Q_{2}$ instead of $\omega_{1}$ and $Q_{1}$. We call $p_{1}$ and $p_{2}$ as "asymmetricity parameters" because the condition of $p_{1}=1$ and $p_{2}=0$ indicates the perfect symmetry of two cavities. Since $\kappa_{m n}$ is less dependent on the cavity structure, it is effective to tune the asymmetricity parameters for minimizing the reflection. Here after, we will show how to choose the structural parameters (and hence, $p_{1}$ and $p_{2}$ ) for minimizing the reflection.

Figure 7 shows the map of the reflection of the all-optical diode as a function of two asymmetricity parameters obtained by NL-CMT for $\kappa_{1}=$ $1.03 \times 10^{-4}, \kappa_{12}=3.75 \times 10^{-5}, \kappa_{21}=3.75 \times 10^{-5}$, and $\kappa_{22}=1.03 \times 10^{-4}$. (The same value of the structure of Fig. 4.) In Fig. 7, for each point ( $p_{1}$ and $\left.p_{2}\right)$, we search the value of $\delta$ which gives the minimum reflection. The reflection is minimum and can be suppressed up to $\sim-120 \mathrm{~dB}$ when the asymmetricity parameters are

$$
\begin{gathered}
p_{1}=\frac{\tau_{2}}{\tau_{1}}=1.90, \\
p_{2}=\tau_{1}\left(\omega_{2}-\omega_{1}\right)=0.58,
\end{gathered}
$$

Resonant wavelength $(\lambda=a / \omega)[\mathrm{nm}]$

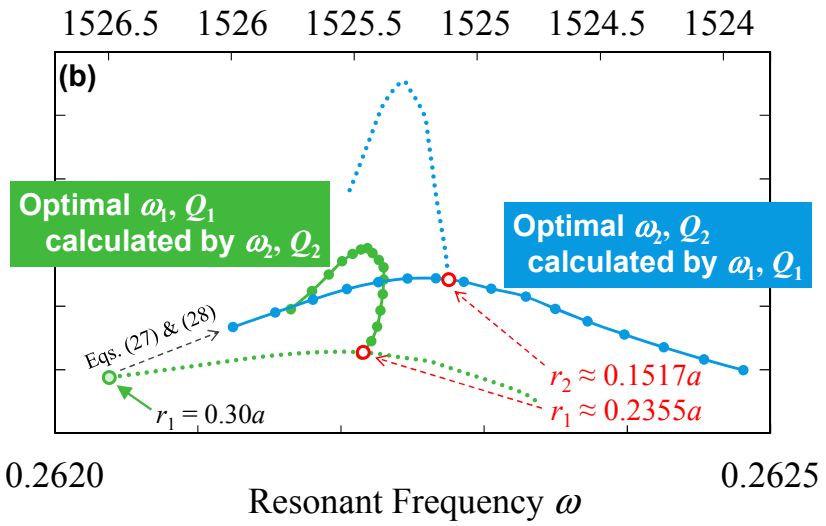

Fig. 8. (a) Structural dependence of resonant frequency and $Q$-value of 1 st and 2nd cavity modes when changing $r_{1}$ and $r_{2}$ between $0.15 a$ and $0.30 a$. The green and blue solid lines corresponds to 1st and 2nd cavity modes, respectively. (b) Optimal resonant frequency and $Q$-value of 1st and 2nd cavity modes. The dotted lines are the same with Fig. 8 (a). The green and blue solid lines represents optimal values calculated by Eqs. (27) and (28). 

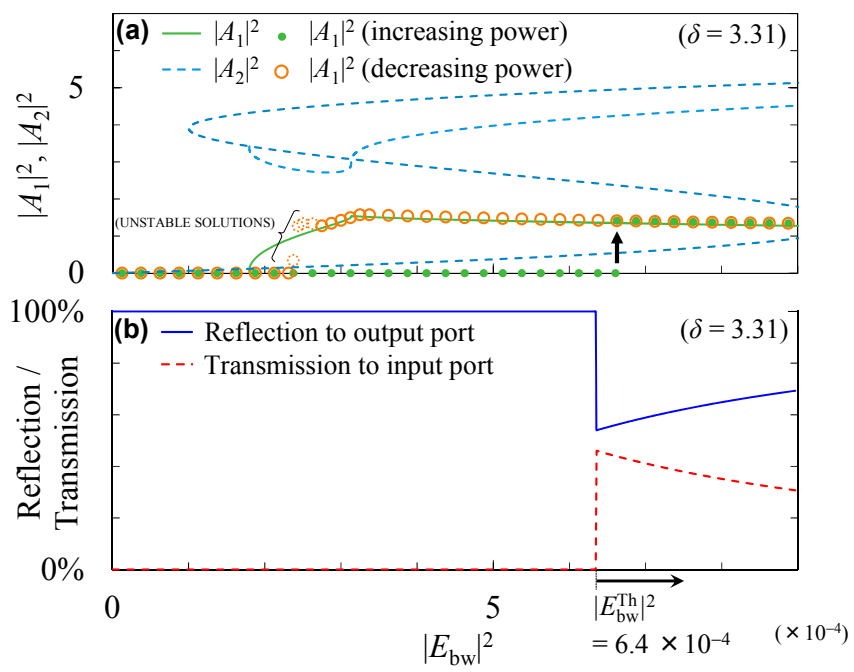

Fig. 9. Resonant characteristics of the all-optical diode with $r_{1}=0.2355 a$ and $r_{2}=0.1517 a$ for the forward propagation ( $\delta=3.31$ ). (a) The amplitude of resonant modes (solid and dashed lines are calculated by Eqs. (8) and (9), and opened and closed circles are calculated by Eqs. (1) and (2) for increasing and decreasing $\left|E_{\text {fw }}\right|$, respectively) and (b) reflection and transmission as a function of input intensity $\left|E_{\mathrm{fw}}\right|^{2}$.

and the detuning parameter is

$$
\delta=\frac{\left(\omega_{1}-\omega\right) \tau_{1}}{2}=3.31 .
$$

Next, we search the structural parameters $r_{1}$ and $r_{2}$ for obtaining the condition of Eqs. (27) and (28). Figure 8(a) shows the structural dependence of the resonant frequency and $Q$-value of 1 st and 2 nd cavity modes when changing $r_{1}$ and $r_{2}$ from $0.15 a$ to $0.30 a$. The green and blue solid lines correspond to 1st and 2nd cavity modes, respectively. The green and blue solid lines in Fig. 8(b) shows the resonant frequency and $Q$-value of 1 st and 2 nd cavity modes satisfying Eqs. (27) and (28). The dotted lines are the same with Fig. 8(a). The solid lines are obtained as follows. For example, if we set $r_{1}=0.30 \mathrm{a}$ for the $1 \mathrm{st}$ cavity, $Q_{1}$ and $\omega_{1}$ are obtained from green dotted lines (left hand side point in this figure). From these $Q_{1}$ and $\omega_{1}, Q_{2}$ and $\omega_{2}$ are calculated from the relationship of Eqs. (27) and (28) (the condition for minimizing reflection obtained by NL-CMT) and these are denoted by blue solid line. The green solid line is obtained as well. Therefore, if we set $r_{1}$, corresponding $Q_{1}$ and $\omega_{1}$ are obtained, and optimum $Q_{2}$ and $\omega_{2}$ can be calculated from Eqs. (27) and (28). The next task is to determine another cavity structure having optimum $Q_{2}$ and $\omega_{2}$. The 2nd cavity structure for obtaining optimum $Q_{2}$ and $\omega_{2}$ is given by the cross point of blue dotted and solid lines. Since the dotted line represents physically possible cavity structure for given geometry (in this case, one $r_{1}$ and two $r$ rods for 1st cavity and one $r_{2}$ and three $r$ rods for 2nd cavity). Therefore, $r_{1}=0.2355 a$ and $r_{2}=0.1517 a$ are the optimum structure for minimizing the reflection (open red circle in Fig. 8(b)), and the corresponding resonant frequency and $Q$-value are $\omega_{1} \approx 0.26222, \omega_{2} \approx$ 0.26228, $Q_{1} \approx 1271$ and $Q_{2} \approx 2414$. Calculated nonlinear coupling coefficients of this structure are $\kappa_{11}=1.03 \times 10^{-4}, \kappa_{12}=3.76 \times 10^{-5}, \kappa_{21}=$ $3.76 \times 10^{-5}$, and $\kappa_{22}=1.03 \times 10^{-4}$.

Figures 9 (a) and (b) show the resonant characteristics of the alloptical diode with $r_{1}=0.2355 a$ and $r_{2}=0.1517 a$ for the forward propagation and the detuning parameter is $\delta=3.31(\omega=0.26187)$. The minimum reflection of $R_{\min } \approx 0 \%(\sim-100 \mathrm{~dB})$ can be obtained in Fig. 9(b) at the input intensity of $\left|E_{\mathrm{fw}}\right|^{2}=3.1 \times 10^{-4}$. Figures 10 (a) and (b) show the resonant characteristics of the all-optical diode with $r_{1}=$ $0.2355 a$ and $r_{2}=0.1517 a$ for the backward propagation. The diode operation is possible within the range of $\left|E_{\mathrm{fw}}\right|^{2}$ from $3.1 \times 10^{-4}$ to $6.4 \times$
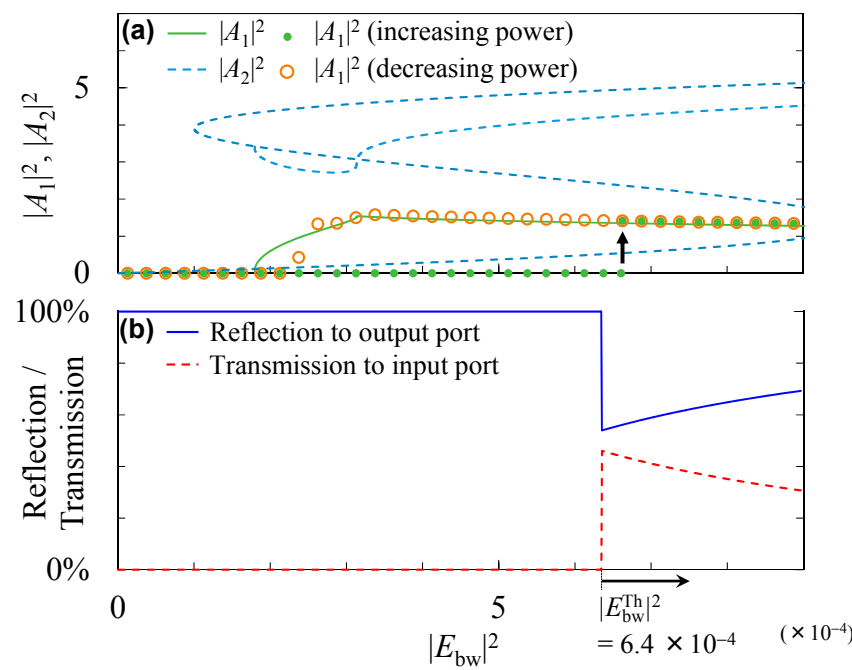

Fig. 10. Resonant characteristics of the all-optical diode with $r_{1}=0.2355 a$ and $r 2=0.1517 a$ for the backward propagation $(\delta=3.31)$. (a) The amplitude of resonant modes (solid and dashed lines are calculated by Eqs. (8) and (9), and opened and closed circles are calculated by Eqs. (1) and (2) for increasing and decreasing $\left|E_{\mathrm{bw}}\right|$, respectively) and (b) reflection and transmission as a function of input intensity $\left|E_{\mathrm{bw}}\right|^{2}$.

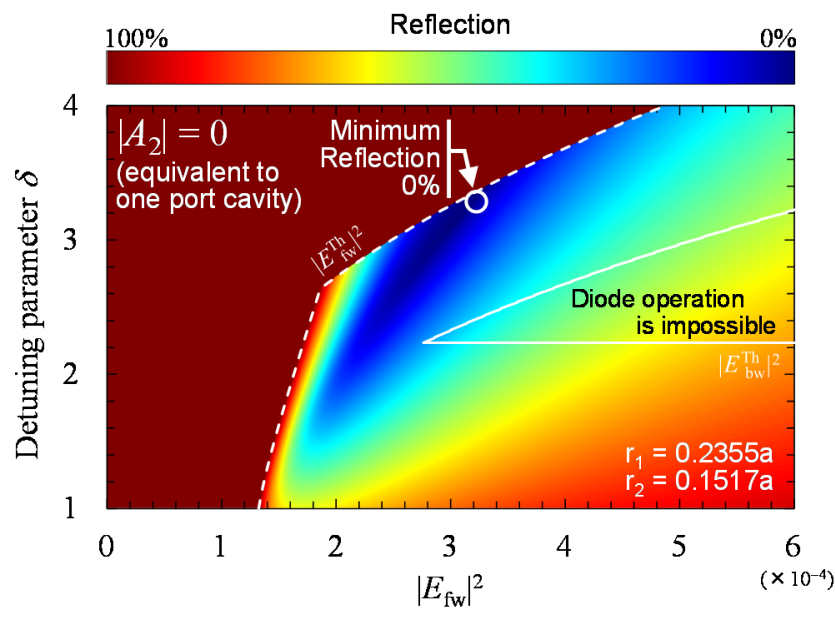

Fig. 11. Map of the reflection of the all-optical diode with $r_{1}=0.2355 a$ and $r_{2}=0.1517 a$ for the forward propagation as a function of input power $\left|E_{\mathrm{fw}}\right|^{2}$ and the detuning parameter $\delta$.

$10^{-4}$. Figure 11 shows the map of the reflection of the all-optical diode with $r_{1}=0.2355 a$ and $r_{2}=0.1517 a$ as a function of input intensity $\left|E_{\mathrm{fw}}\right|^{2}$ and the detuning parameter $\delta$ The drastic reduction of the reflection can be seen compared with Fig. 6 . Also, since the area of the low-reflection region is very wide, the designed structure has wide operation range, leading to strong tolerance to the operation condition.

\section{Nonlinear Analysis of NL-FETD-BPM}

Here, we confirm the validity of the discussion by comparing the results obtained by NL-CMT and rigorous numerical NL-FETD-BPM.

Figures 12 (a) and (b) show the reflection characteristics as a function of the frequency and power of input lightwave obtained by NLCMT (dashed line) and NL-FETD-BPM (dots). For Fig. 12 (a), the input power is constant $(0.270 \mathrm{~W} / \mu \mathrm{m})$, for $(\mathrm{b})$, the wavelength is constant $(1527.46 \mathrm{~nm})$. The input power of $0.270 \mathrm{~W} / \mu \mathrm{m}$ in the NL-FETD-BPM scheme is corresponding to $\left|E_{\mathrm{fw}}\right|^{2}=3.2 \times 10^{-4}$ in the NL-CMT scheme. If the height of the rod is $a(=400 \mathrm{~nm})$, input power and power density are 

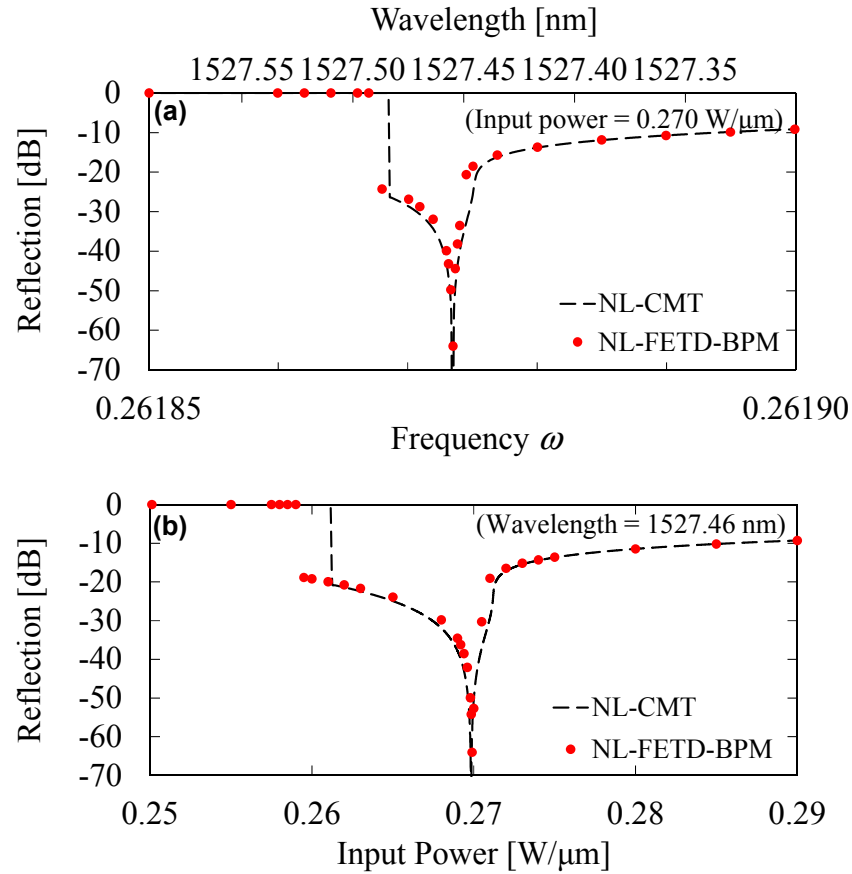

Fig. 12. Reflection characteristics as a function of (a) frequency for input power of $0.270 \mathrm{~W} / \mu \mathrm{m}$ and (b) input power for input wavelength of $1527.46 \mathrm{~nm}$.

approximately $0.108 \mathrm{~W}(=0.270 a)$ and $0.675 \mathrm{~W} / \mu \mathrm{m}^{2}(=0.270 / a)$. The solution of NL-FETD-BPM agrees well with that of NL-CMT except for the small difference in threshold power between two solutions. The reflection of $-65 \mathrm{~dB}$ is obtained for NL-FETD-BPM, showing the effectiveness of the proposed strategy for suppressing the reflection calculated by NL-CMT. Under this input condition, the maximum refractive index change is approximately 0.01 .

Figures 13 (a) and (b) show the forward and backward transmission as a function of (a) frequency and (b) power of the input lightwave calculated by NL-FETD-BPM. The diode operation is possible within the range of input wavelength from $1526.91 \mathrm{~nm}$ to $1527.49 \mathrm{~nm}$ for input power of $0.270 \mathrm{~W} / \mu \mathrm{m}$ as shown in Fig. 13 (a), and the range of input power from $0.26 \mathrm{~W} / \mu \mathrm{m}$ to $0.53 \mathrm{~W} / \mu \mathrm{m}$ for input wavelength of 1527.46 $\mathrm{nm}$ as shown in Fig. 13 (b). We can see that the NTR is almost $90 \mathrm{~dB}$ in the diode operation region, which is extremely large value.

\section{CONCLUSION}

The all-optical diode based on the asymmetric L-shaped nonlinear PC cavity is investigated by the NL-CMT and the design method to suppress the reflection in the diode operation is illustrated. For designing the reflection-suppressed all-optical diode, only two "asymmetricity parameters" are crucial values. In our proposed strategy, it is required to specify the asymmetricity parameters by using NL-CMT and calculate the resonant frequency and $Q$-value by using FETD-BPM. This simple procedure enables to design the reflection-suppressed structure in a very short calculation time. Furthermore, we confirm that the designed all-optical diode achieves the very small reflection $(-65 \mathrm{~dB})$ as well as extremely large NTR $(90 \mathrm{~dB})$.

\section{REFERENCES}

1. D. Jalas, A. Petrov, M. Eich, W. Freude, S. Fan, Z. Yu, R. Baets, M. Popović, A. Melloni, J. D. Joannopoulos, M. Vanwolleghem, C. R. Doerr, and H. Renner, "What is - and what is not - an optical isolator," Nat. Photonics 7, 579-582 (2013).
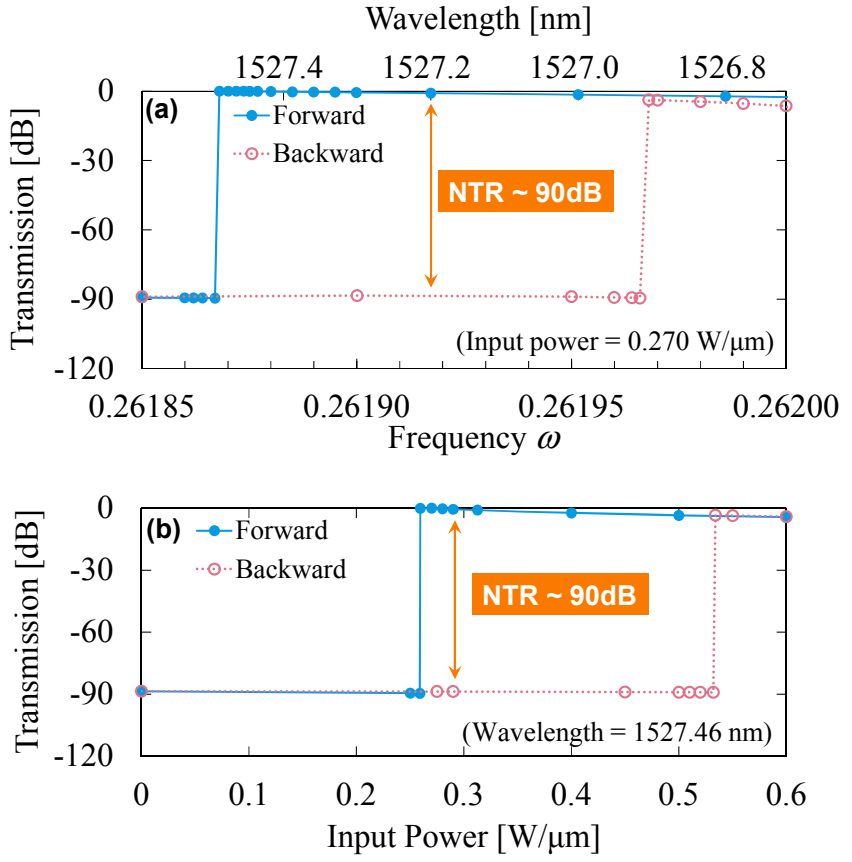

Fig. 13. Forward and backward transmission as a function of (a) frequency for input power of $0.270 \mathrm{~W} / \mu \mathrm{m}$ and (b) input power for input wavelength of $1527.46 \mathrm{~nm}$.

2. A. B. Khanikaev and A. Alù, "Optical isolators: Nonlinear dynamic reciprocity," Nat. Photonics 9, 359-361 (2015).

3. Y. Shi, Z. Yu, and S. Fan, "Limitations of nonlinear optical isolators due to dynamic reciprocity," Nat. Photonics 9, 388-392 (2015).

4. L. Bi, J. Hu, P. Jiang, D. H. Kim, G. F. Dionne, L. C. Kimerling, and C. A. Ross, "On-chip optical isolation in monolithically integrated non-reciprocal optical resonators," Nat. Photonics 5, 758-762 (2011).

5. B. J. H. Stadler and T. Mizumoto, "Integrated magneto-optical materials and isolators: a review," IEEE Photonics J. 6, 0600215-1-0600215-16 (2014).

6. K. Gallo, G. Assanto, K. R. Parameswaran, and M. M. Fejer, "All-optical diode in a periodically poled lithium niobate waveguide," Appl. Phys. Lett. 79, 314 (2001).

7. S. F. Mingaleev and Y. S. Kivshar, "Nonlinear transmission and light localization in photonic-crystal waveguides," J. Opt. Soc. Am. B 19, 22412249 (2002)

8. X.-S. Lin, W.-Q. Wu, H. Zhou, K.-F. Zhou, and S. Lan, "Enhancement of unidirectional transmission through the coupling of nonlinear photonic crystal defects," Opt. Express 14, 2429-2439 (2006).

9. X.-S. Lin, J.-H. Yan, L.-J. Wu, and S. Lan, "High transmission contrast for single resonator based all-optical diodes with pump-assisting," Opt. Express 16, 20949-20954 (2008).

10. X. Huang and S. Fan, "Complete all-optical silica fiber isolator via stimulated Brillouin scattering," J. Lightw. Technol. 29, 2267-2275 (2011).

11. W. Ding, B. Luk'yanchuk, and C.-W. Qiu, "Ultrahigh-contrast-ratio silicon Fano diode," Phys. Rev. A 85, 025806-1-025806-5 (2012).

12. L. Fan, J. Wang, L. T. Varghese, H. Shen, B. Niu, Y. Xuan, A. M. Weiner, and M. Qi, "An all-silicon passive optical diode," Science 335, 447-450 (2012).

13. J. Wang, L. Fan, L. T. Varghese, H. Shen, Y. Xuan, B. Niu, and M. Qi, "A theoretical model for an optical diode built with nonlinear silicon microrings," J. Lightw. Technol. 31, 313-321 (2013).

14. K. Saha, Y. Okawachi, O. Kuzucu, M. Menard, M. Lipson, and A. L. Gaeta, "Chip-scale broadband optical isolation via bragg scattering Four-Wave Mixing," in CLEO: 2013, OSA Technical Digest (online) (Optical Society of America, 2013) (OSA, 2013), p. QF1D.2.

15. E. N. Bulgakov and A. F. Sadreev, "All-optical diode based on dipole modes of Kerr microcavity in asymmetric L-shaped photonic crystal waveguide," Opt. Lett. 39, 1787-1790 (2014). 
16. L. Fan, L. T. Varghese, J. Wang, Y. Xuan, A. M. Weiner, and M. Qi, "Silicon optical diode with 40 dB nonreciprocal transmission," Opt. Lett. 38, 12591261 (2013).

17. M. Koshiba, Y. Tsuji, and M. Hikari, "Time-domain beam propagation method and its application to photonic crystal circuits," J. Lightw. Technol. 18, 102-110 (2000).

18. T. Fujisawa and M. Koshiba, "Time-domain beam propagation method for nonlinear optical propagation analysis and its application to photonic crystal circuits," J. Lightw. Technol. 22, 684-691 (2004).

19. E. N. Bulgakov, K. Pichugin, and A. F. Sadreev, "Symmetry breaking for transmission in a photonic waveguide coupled with two off-channel nonlinear defects," Phys. Rev. B 83, 045109-1-045109-14 (2011).

20. E. N. Bulgakov and A. F. Sadreev, "All-optical manipulation of light in X-and T-shaped photonic crystal waveguides with a nonlinear dipole defect," Phys. Rev. B 86, 075125-1-075125-10 (2012).

21. M. Soljačić, M. Ibanescu, S. G. Johnson, Y. Fink, and J. D. Joannopoulos, "Optimal bistable switching in nonlinear photonic crystals," Phys. Rev. E 66, 055601-1-055601-4 (2002).

22. R. L. Sutherland, Handbook of Nonlinear Optics, 2nd editio (Marcel Dekker, 2003).

23. W. C. Hurlbut, K. L. Vodopyanov, P. S. Kuo, M. M. Fejer, and Y. S. Lee, "Multi-photon absorption and nonlinear refraction of GaAs in the midinfrared," Conf. Lasers Electro-Optics 2006 Quantum Electron. Laser Sci. Conf. CLEO/QELS 2006 32, 668-670 (2006). 